\title{
Bright metal-poor variables: Why "Anomalous" Cepheids?
}

\author{
F. Caputo ${ }^{1}$, V. Castellani ${ }^{1,2}$, S. Degl'Innocenti ${ }^{3,4}$, G. Fiorentino $^{1,5}$, and M. Marconi ${ }^{6}$ \\ 1 INAF - Osservatorio Astronomico di Roma, Via di Frascati 33, 00040 Monteporzio Catone, Italy \\ 2 INFN, Sezione di Ferrara, via Paradiso 12, 44100 \\ 3 Dipartimento di Fisica, Universita' di Pisa, via Buonarroti 2, 56127 Pisa, Italy \\ ${ }^{4}$ INFN, Sezione di Pisa, via Buonarroti 2, 56127 Pisa, Italy \\ 5 Universita' "Tor Vergata”, via della Ricerca Scientifica 1, 00133, Roma, Italy \\ ${ }^{6}$ INAF Osservatorio Astronomico di Capodimonte, Via Moiariello 16, 80131 Napoli, Italy
}

Received 20 February 2004 / Accepted 18 May 2004

\begin{abstract}
We investigate the theoretical scenario concerning the large sample of variables recently discovered in the dwarf, metal-poor irregular galaxy Leo A, focusing the attention on the "Anomalous" Cepheid phenomenon and its correlation with RR Lyrae stars, Classical and Population II Cepheids. To this purpose, we make use of suitable stellar and pulsation models to depict the pulsational history of evolutionary structures with metallicity $Z=0.0004$. We find that He-burning pulsators are expected only outside the mass interval $\sim 0.8-1.7 M_{\odot}$. Stars from $\sim 1.8$ to $4 M_{\odot}$, a mass range including both Anomalous and Classical Cepheids, populate to good approximation a common $M_{V}-\log P_{\mathrm{F}}$ instability strip, independently of the previous occurrence of a He flash event. Their periods and luminosities increase with the stellar mass and they are at a lower luminosity level $M_{V, \mathrm{LE}} \sim-0.5 \mathrm{mag}$, as observed in Leo A. The class of less massive pulsators $\left(M<0.8 M_{\odot}\right.$, namely $R R$ Lyrae stars and Population II Cepheids) populate a distinct instability strip, where the magnitudes become brighter and the periods longer when the pulsator mass decreases. The dependence on metal content in this scenario has been investigated over the range $Z=0.0002$ to 0.008 . The edges of the pulsational strip for the more massive class of pulsators appear to be independent of metallicity, but with a minimum mass of these bright pulsators which decreases with decreasing metallicity, thus decreasing the predicted minimum luminosity and period. Comparison with data for Cepheids in Leo A and in the moderately metal-rich extragalactic stellar system Sextans A discloses an encouraging agreement with the predicted pulsational scenario. On this basis, we predict that in a stellar system where both RR Lyrae stars and Cepheids are observed their magnitude difference may help in constraining both the metal content and the distance. The current classification of metal-poor Cepheids is briefly discussed and suggestion is advanced for an updated terminology abreast with the current knowledge of stellar evolution.
\end{abstract}

Key words. stars: variables: Cepheids - stars: evolution

\section{Introduction}

Even though the majority of metal-poor radial pulsators belongs to the RR Lyrae class, other kinds of variables are observed both in Galactic globular clusters and in extragalactic metal-poor stellar systems. According to the current nomenclature, among the objects brighter than RR Lyrae stars one finds Population II Cepheids (P2C) and Anomalous Cepheids (AC), the former with periods of from $\sim 1$ to $\sim 25$ days, the latter from $\sim 0.3$ to $\sim 2$ days. Both types of variables are interpreted in terms of central He-burning structures, as are RR Lyrae stars, but as being either less massive (P2Cs) or more massive ( $\mathrm{ACs}$ ) than RR Lyrae stars with similar metal content.

Investigations based on the predictions of stellar evolution and radial pulsation models have already shown that P2Cs originate from hot, low-mass Zero Age Horizontal Branch (ZAHB) stars evolving towards the Asymptotic Giant Branch (AGB) and crossing the instability strip at a luminosity larger than the RR Lyrae level (see, e.g., Bono et al. 1997a, and references therein, as well as the recent review by Wallerstein 2002). Since the pulsation period $P$ increases with increasing luminosity and/or with decreasing mass, these bright low mass pulsators are predicted to have longer periods than RR Lyrae stars, as is observed.

Concerning ACs, it has been shown that at metal contents $Z \leq 0.0004$ and for not-too-old ages ( $\leq 2 \mathrm{Gyr}$ ) the effective temperature in ZAHB models, which normally decreases with increasing mass, reaches a minimum at $\log T_{\mathrm{e}} \sim 3.74$ $(Z=0.0001)$ or $\sim 3.72(Z=0.0004)$ around $1-1.2 M_{\odot}$ (see Castellani \& Degl'Innocenti 1995; Caputo \& Degl'Innocenti 1995 and references therein). By further increasing the mass above this value, both the luminosity and the effective temperature of the ZAHB structure increase, producing a "ZAHB turnover" and an "upper horizontal branch" which intersects the instability strip again at a luminosity larger than the RR Lyrae level (see Bono et al. 1997b). In this case the effect of the higher luminosities is somehow "balanced" by the larger masses, and consequently these bright massive pulsators will show periods 
that are not significantly longer than those typical of RR Lyrae stars, in agreement with the observed behavior for ACs. On this simple basis, one can understand why ACs and P2Cs appear to obey distinctive Period-Luminosity relations and why, at a given luminosity, ACs have shorter periods than P2Cs. It turns out that, at a given period, ACs appear more luminous than P2Cs, a feature which is at the origin of their supposed "anomaly".

ACs are very rare in globular clusters, whereas they have been found in all dwarf spheroidal galaxies that have been surveyed for variable stars (see, e.g., Mateo 1998; Siegel \& Majewski 2000; Pritzl et al. 2002; Da Costa et al. 2002; Dall'Ora et al. 2003; Baldacci et al. 2004). Recently, Dolphin et al. (2002, hereafter D02) reported the results of a search for short-period ( $P \leq 2$ days) variables in Leo A, a Local Group dwarf irregular galaxy characterized by a very low metal abundance $(Z \sim 0.0004)$. According to D02, eight candidate RR Lyrae stars were found, with a mean magnitude of $\langle V\rangle=25.10 \pm 0.09 \mathrm{mag}$, suggesting the presence in this galaxy of an old ( $\sim 10-11$ Gyr) stellar population. The same study reports the discovery of several (84) variables brighter than $V=$ $24.5 \mathrm{mag}$ and with periods between $\sim 0.4$ and 2 days. Although these properties correspond to the well-recognized behavior of ACs, the authors suggest that, rather than being indicative of this class of variables, they appear as a natural extension to low metallicity of "classical" (i.e. metal-intermediate) shortperiod Cepheids, such as those observed in the Large and Small Magellanic Clouds ( $Z \sim 0.008$ and $\sim 0.004$, respectively).

In this paper we will show that ACs indeed represent indeed the natural extension of classical Cepheids to lower metallicities and masses. To this end, in the following we will rely on suitable sets of stellar evolution and pulsation models to discuss the "Anomalous" Cepheid phenomenon and its correlation with RR Lyrae stars, Population II and Classical Cepheids. The theoretical scenario is presented in Sect. 2, while Sect. 3 discusses the predicted Period-Luminosity distribution when $Z=0.0004$. Section 4 deals with the dependence on the assumed metal content and the comparison with observed data is given in Sect. 5. As a concluding remark, in the last section we direct the reader's attention to the evidence that the current classification might be misleading in some respects, perhaps requiring an updated terminology based on the current improved knowledge of stellar evolution.

\section{The theoretical scenario}

Radial pulsation is a phenomenon appearing only in selected evolutionary phases. Given a metal abundance, for each given stellar mass and luminosity there is a maximum (blue edge) and a minimum effective temperature (red edge) for the onset of the pulsation instability. If the luminosity is varied, for each given mass one has the so called "instability strip" which crosses the HR diagram from the higher temperatures and lower luminosities towards lower temperatures and higher luminosities. If and when the evolving star intersects "its" mass-dependent instability strip, it starts pulsating with a period strictly constrained by its mass, luminosity, and effective temperature.
With respect to static stars, radial pulsating structures present the undeniable advantage of at least one additional observable, as given by the pulsation period, which, besides being unaffected by distance and reddening, yields relevant constraints to the stellar structural parameters. Combining evolution and pulsation models one can predict the behavior of radial pulsators occurring in the different evolutionary phases, providing a theoretical frame for a sound interpretation of the different classes of observed variables. In this way, we have recently presented a detailed analysis of RR Lyrae stars in the Galactic globular cluster M 3 (see Marconi et al. 2003).

To discuss anomalous and classical Cepheids in Leo A, we will make use of the pulsation models with $Z=0.0001$ and 0.0004 recently computed by Marconi et al. (2004) for masses in the range 1.3-2.2 $M_{\odot}$ and luminosities $\log L / L_{\odot}=$ 1.82 to 2.28 , implemented with a set of $4 M_{\odot}$ models at $Z=$ 0.0004 and $\log L / L_{\odot}=3.5$. By comparison with similar models presented by Bono et al. (2002) for $Z=0.008$ over the mass range $3-5 M_{\odot}$, one finds that all over the whole range $Z=0.0001$ to 0.008 the blue (FOBE) and red (FRE) edges of the instability strip follow the two relations

$$
\begin{aligned}
\log T_{\mathrm{e}}(\mathrm{FOBE})= & 3.925( \pm 0.008)-0.052 \log L \\
& +0.042 \log M-0.006 \log Z \\
\log T_{\mathrm{e}}(\mathrm{FRE})= & 3.876( \pm 0.008)-0.065 \log L \\
+ & 0.058 \log M-0.006 \log Z
\end{aligned}
$$

where $M$ and $L$ are in solar units, whereas the period of fundamental $(\mathrm{F})$ pulsators is given by:

$$
\begin{aligned}
\log P_{\mathrm{F}}= & 10.925( \pm 0.005)+0.818 \log L \\
& -0.616 \log M-3.309 \log T_{\mathrm{e}}+0.012 \log Z .
\end{aligned}
$$

Moreover, in order to compare the predicted behavior of these relatively massive pulsators with that of the less massive RR Lyrae and P2C variables, pulsation models with metal content $Z=0.0001-0.006, M=0.58-0.80 M_{\odot}$ and $\log L / L_{\odot}=$ 1.51 to 1.91 have been considered, for which it has already been found (Marconi et al. 2003; Di Criscienzo et al. 2004) that

$$
\begin{aligned}
\log T_{\mathrm{e}}(\mathrm{FOBE})= & 3.970( \pm 0.005)-0.057 \log L \\
& +0.094 \log M \\
\log T_{\mathrm{e}}(\mathrm{FRE})= & 3.957( \pm 0.010)-0.102 \log L \\
+ & 0.073 \log M
\end{aligned}
$$

$\log P_{\mathrm{F}}=11.039( \pm 0.005)+0.833 \log L-0.651 \log M$

$$
-3.350 \log T_{\mathrm{e}}+0.008 \log Z \text {. }
$$

In all cases one finds that first-overtone (FO) pulsators can be "fundamentalised" by adopting $\log P_{\mathrm{F}}=\log P_{\mathrm{FO}}+0.13$.

All the pulsation models we are referring to rely on the use of the same nonlinear convective hydrodynamical code, an approach which is of central importance to gain reliable information on the boundaries for fundamental or first overtone pulsation. The inadequacy of linear computations in this respect has already been discussed by Bono et al. (1999). However, here we notice that even in the linear approach (see, e.g., Chiosi et al. 1993), 


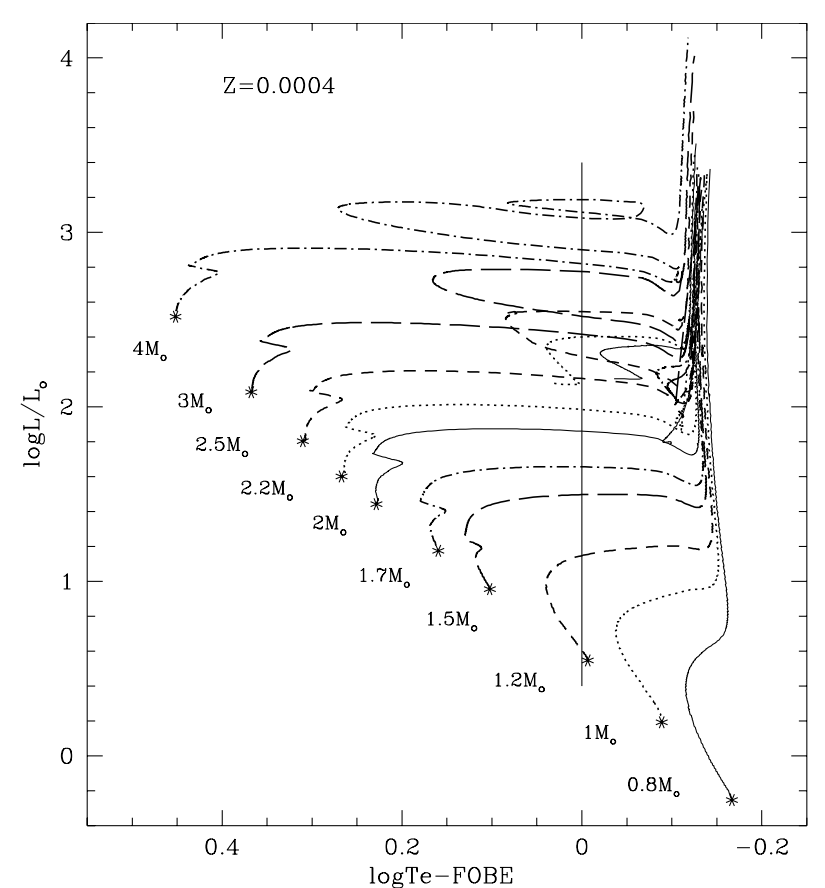

Fig. 1. Evolutionary tracks from Main Sequence to Asymptotic Giant Branch at $Z=0.0004$ and for selected stellar masses. The effective temperature is scaled to the predicted red edge (FRE) of the pulsation region. Asterisks mark the MS models for the various labeled masses.

one predicts a significant dependence on the pulsator structural parameters (mass and luminosity), at variance with the hypothesis of a constant value $\left(\log T_{\mathrm{e}}(\mathrm{FRE})=3.73\right)$ as recently adopted by Cordier et al. (2003).

Such a pulsational scenario has been combined with canonical (i.e., no mass-loss, no overshooting) evolutionary models with $Z=0.0004$ from the "Pisa Evolutionary Library" (http://astro.df.unipi.it/SAA/PEL/ZO.html). All models cover the major phases of both $\mathrm{H}$ and He burning (see Cariulo et al. 2004 for details). Following the procedure presented by Bono et al. (1997a,b) and Marconi et al. (2003), one can depict the pulsational history of each evolutionary model (i.e., for each given mass and original composition) by investigating the evolutionary paths in terms of the difference between the actual effective temperature and the predicted values at FRE and FOBE at the various luminosities.

The straightforward consequence of this procedure is shown in Figs. 1 and 2, where evolutionary sequences for masses 0.8 to $4.0 M_{\odot}$ are plotted in a "pulsational" HR diagram, where effective temperatures are scaled, for each mass and luminosity, to the appropriate FRE and FOBE, respectively. For each given mass the predicted pulsators are given by models with an effective temperature falling between the blue and red edges of the pulsation region (FOBE $\geq \log T_{\mathrm{e}} \geq \mathrm{FRE}$ ).

Inspection of Figs. 1 and 2 provides a general overview of the connection between pulsation and evolution. In particular, varying the mass, one derives that:

1. for masses smaller than $\sim 1.0 M_{\odot}$ the whole evolution proceeds at an effective temperature lower than FRE, and no variables are expected, unless mass-loss is driving the structures into the RR Lyrae instability region (see later);

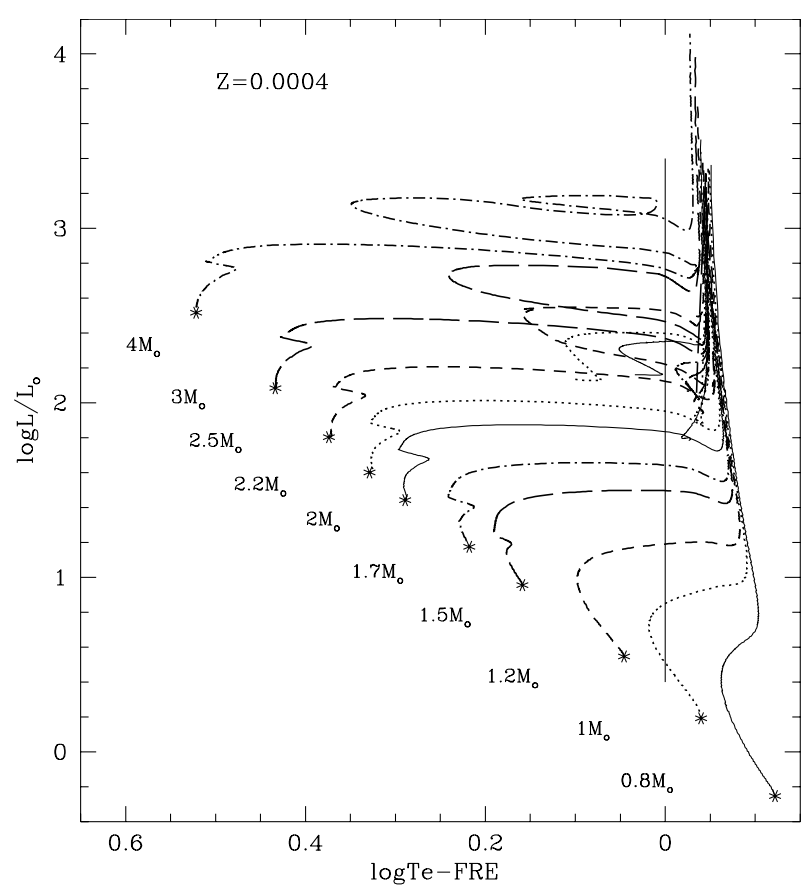

Fig. 2. As in Fig. 1, but with the effective temperature scaled to the predicted blue edge (FOBE) of the pulsation region.

2. for masses from $\sim 1.0 M_{\odot}$ to $\sim 1.7 M_{\odot}$ the effective temperature of He-burning models ("blue loops") is cooler than FRE, so that one would expect variables only during the short-lived H-burning phase when the stars evolve redward after central hydrogen exhaustion;

3. for evolving stars with masses larger than $1.7 M_{\odot}$ crossing of the instability strip occurs both in the phase following the exhaustion of central hydrogen and during central Heburning.

Thus, if mass loss is neglected, central He-burning pulsators with $Z=0.0004$ are expected only from stars more massive than $1.7 M_{\odot}$ and brighter than $\log L / L_{\odot} \sim 2.15$.

The effect of mass loss and the consequent appearance of low-mass central He-burning variables (i.e. RR Lyrae stars and P2Cs) deserve further comments. For masses $M>1.0 M_{\odot}$ the effects of mass loss are substantially irrelevant: even a loss of $0.3 M_{\odot}$ by a $1.7 M_{\odot}$ progenitor $\left(M_{\mathrm{c}}=0.467 M_{\odot}\right)$ will yield a $1.4 M_{\odot}$ He-burning structure which is only slightly fainter than the $1.4 M_{\odot}$ model without mass loss $\left(M_{\mathrm{c}}=0.477 M_{\odot}\right.$, see Castellani \& Degl'Innocenti (1995) for details) In contrast, a mass loss of $\sim 0.1-0.2 M_{\odot}$ in less massive progenitors has dramatic consequences, moving the newly born He-burning structures to large effective temperatures, along the ZAHB locus, so that they will successively cross the instability strip.

All this is summarized in Fig. 3, where we present an expanded portion of Fig. 2 showing the post He-flash, central He-burning paths of structures with masses from 2.0 to $0.8 M_{\odot}$ in the absence of mass loss (solid lines), compared with the predicted boundaries of the instability region (vertical lines). The same figure also shows the evolution from the Zero Age Horizontal Branch (ZAHB: dashed line) of structures 


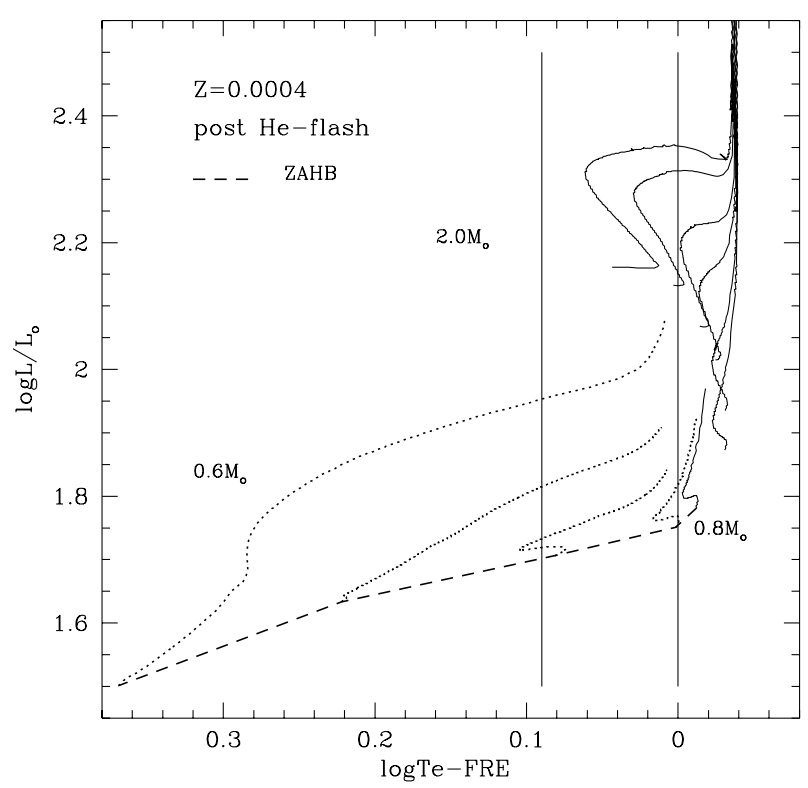

Fig. 3. Enlarged portion of Fig. 3 showing the post He-flash, central He-burning evolutionary tracks of stellar structures with mass $0.8,1.0$, $1.2,1.5,1.7,1.9$ and $2.0 M_{\odot}$ in the absence of mass loss during the RGB phase (solid lines). The dotted lines depict the evolution from the ZAHB (dashed line) of models with mass $0.60,0.65,0.70$ and $0.75 M_{\odot}$ originating from a $0.8 M_{\odot}$ progenitor.

with mass $0.6-0.75 M_{\odot}($ dotted line $)$ originated from a $0.8 M_{\odot}$ progenitor.

Inspection of the figure shows that, as expected, the predicted luminosity of low mass $\left(<0.8 M_{\odot}\right)$ pulsators increases with decreasing mass, at variance with the mass-luminosity relation followed by the more massive $\left(>1.7 M_{\odot}\right)$ structures. As a conclusion, we can expect that

a) with $Z=0.0004$ no central He-burning pulsators are expected in the mass range $\sim 0.8-1.7 M_{\odot}$;

b) outside this forbidden range, more massive or less massive pulsators should follow distinctive period-luminosity relations as a consequence of their opposite mass-luminosity relations.

\section{The period-luminosity distribution}

By selecting the models falling within their instability strip, one can now derive the predicted period-magnitude diagram of pulsators with $Z=0.0004$ and various masses. To this purpose, we used bolometric corrections by Castelli et al. (1997) to get absolute visual magnitudes, while fundamental periods $P_{\mathrm{F}}$ are estimated for each given mass, luminosity and effective temperature by means of the relations given in the previous section.

Figure 4 shows the predicted $M_{V}-\log P_{\mathrm{F}}$ diagram of central He-burning pulsators at the labeled masses. Stars from 1.9 to $4 M_{\odot}$ undergoing their radial pulsation phase are found to define a nearly unique instability strip, independently of the occurrence of a quiet $\left(>2.1 M_{\odot}\right)$ or a flashing $\left(\leq 2.1 M_{\odot}\right) \mathrm{He}$ ignition. With increasing mass the pulsators become generally brighter and have longer periods. As shown in the figure, for these massive central He-burning pulsators with $Z=0.0004$ one can predict a lower luminosity limit of $M_{V, \mathrm{LE}} \sim-0.5 \mathrm{mag}$,

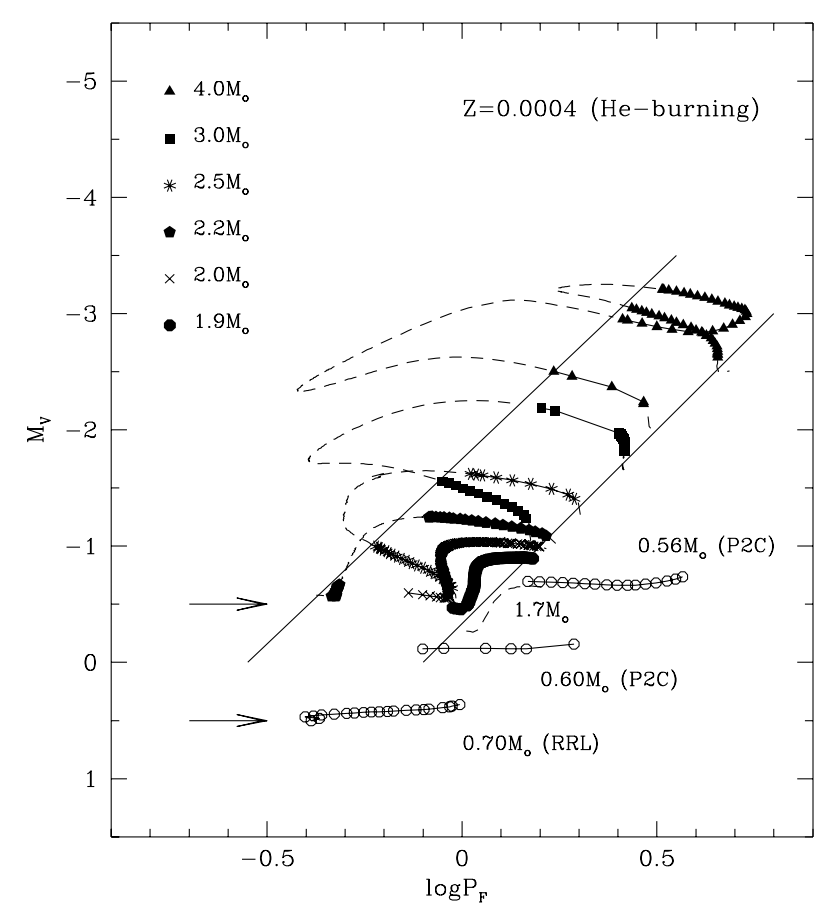

Fig. 4. Predicted absolute visual magnitude $M_{V}$ versus period $P_{\mathrm{F}}$ for He-burning fundamental pulsators with $Z=0.0004$ and selected masses, in the absence of mass-loss (solid symbols). The portion of the tracks outside the pulsation region is drawn with a dashed line. The solid lines show the edges of the predicted $M_{V}-\log P_{\mathrm{F}}$ distribution, while the upper arrow marks the predicted absolute magnitude $M_{V, \mathrm{LE}} \sim-0.5 \mathrm{mag}$ of the lower envelope of the pulsator distribution. Open symbols illustrate the behavior of the predicted RR Lyrae (RRL) and Population II Cepheids (P2C) as caused by efficient mass loss. The lower arrow shows the predicted absolute magnitude of RR Lyrae stars.

as well as fundamental periods as short as $\log P_{\mathrm{F}} \sim-0.3$. Concerning less massive pulsators evolving off their ZAHB $\left(M<0.8 M_{\odot}\right.$, see Fig. 3), Fig. 4 shows that they populate a distinct instability strip, where the magnitude becomes brighter and the period longer with decreasing pulsator mass.

Inspection the range of predicted (fundamental) periods and magnitudes, in the pulsators with mass $\sim 1.9-2.2 M_{\odot}$ one can easily recognize typical features of observed ACs in dwarf spheroidal galaxies (see Marconi et al. 2004), while the $0.7 M_{\odot}$ and less massive pulsators should represent RR Lyrae stars and Population II Cepheids, respectively. Note that the predicted luminosity of RR Lyrae stars $\left(M_{V}(\mathrm{RRL}) \sim 0.5 \mathrm{mag}\right)$ is only $\sim 1$ mag fainter than the lower envelope of the massive pulsator distribution $\left(M_{V, \mathrm{LE}} \sim-0.5 \mathrm{mag}\right)$, whereas the visual magnitude of the predicted P2C pulsators can be even brighter than $M_{V, \mathrm{LE}}$. However, at a given period one finds ACs brighter than P2C stars, as observed. As a whole, the results plotted in Fig. 4 disclose that post-He flash structures with mass $\sim 1.9$ $2.2 M_{\odot}$ (i.e., AC candidates) are the natural extension to fainter magnitudes of the more massive central He-burning pulsators of similar metal content. 


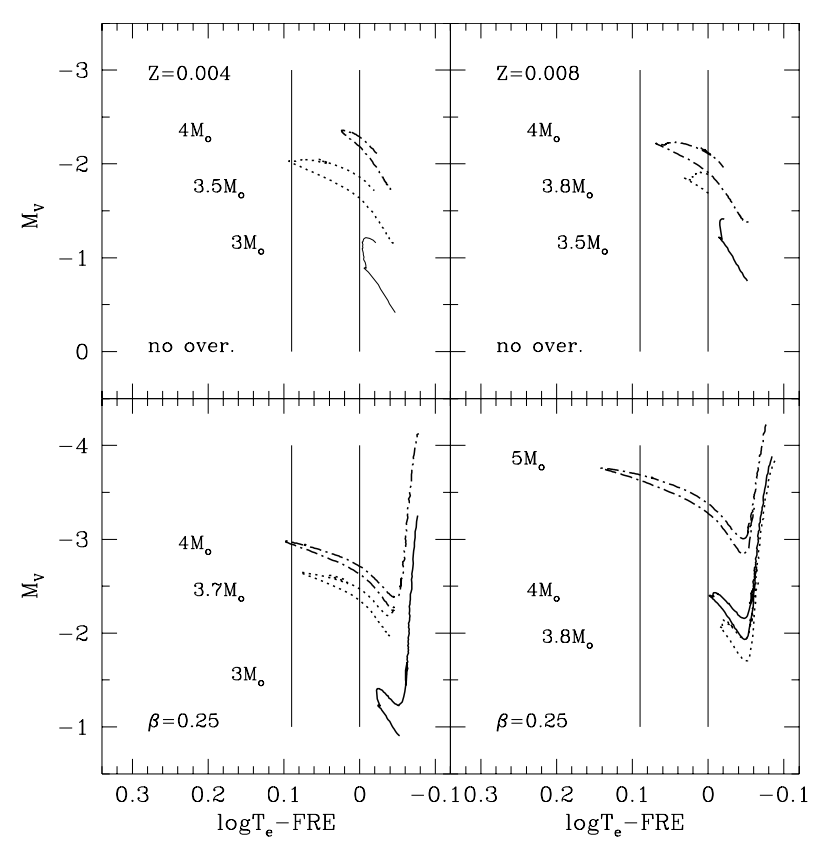

Fig. 5. Central He-burning tracks without (upper panels) and with overshooting (lower panels) at the labeled metal content. The effective temperatures are scaled to the predicted FRE. The solid lines depict the pulsation region.

\section{The effect of metallicity}

The effect of metallicity on the pulsational scenario has been investigated by repeating the above procedure but for the selected metallicity values $Z=0.0002,0.0006,0.001,0.004$ and 0.008 , again using canonical evolutionary tracks from the Pisa Evolutionary Library. As an example, the upper panels in Fig. 5 show that the minimum mass for the occurrence of massive central He-burning pulsators changes from $1.9 M_{\odot}$ ( $Z=0.0004)$ to $\sim 3.0 M_{\odot}$ if $Z=0.004$ and to $3.6 M_{\odot}$ when $Z=0.008$. Correspondingly the lower luminosity level increases from $M_{V} \sim-0.5 \mathrm{mag}$ to $M_{V} \sim-1.3 \mathrm{mag}(Z=0.004)$ and $M_{V} \sim-1.5(Z=0.008)$.

The lower panels in the same figure show that the adoption of stellar models with overshooting $(\beta=0.25)$ yields larger values for the minimum mass, and consequently brighter magnitudes. One can estimate $\sim 3.3 M_{\odot}$ at $M_{V} \sim-1.7$ and $\sim 4.0 M_{\odot}$ at $M_{V} \sim-2.4 \mathrm{mag}$, with $Z=0.004$ and 0.08 , respectively. Note that, since at such metal contents $M_{\mathrm{fl}}<2.3 M_{\odot}$ (see Cassisi et al. 1997), all the massive pulsators undergo a quiet ignition of central He-burning.

Figure 6 shows that the predicted period-luminosity distribution of these more metal-rich pulsators appears in good agreement with the edges of the instability strip at $Z=0.0004$ (solid lines); the only difference is that the faintest magnitudes are significantly brighter than the predicted value for $Z=0.0004$ (upper arrow in each panel), with the shortest periods increasing well above the $Z=0.0004$ value $\left(\log P_{\mathrm{F}}=-0.3\right.$, see Fig. 4). Moreover, since the luminosity level of RR Lyrae stars (dotted lines) is even fainter than at $Z=0.0004$ (lower arrow in each panel), the difference in magnitude between the faintest massive pulsators and RR Lyrae variables becomes

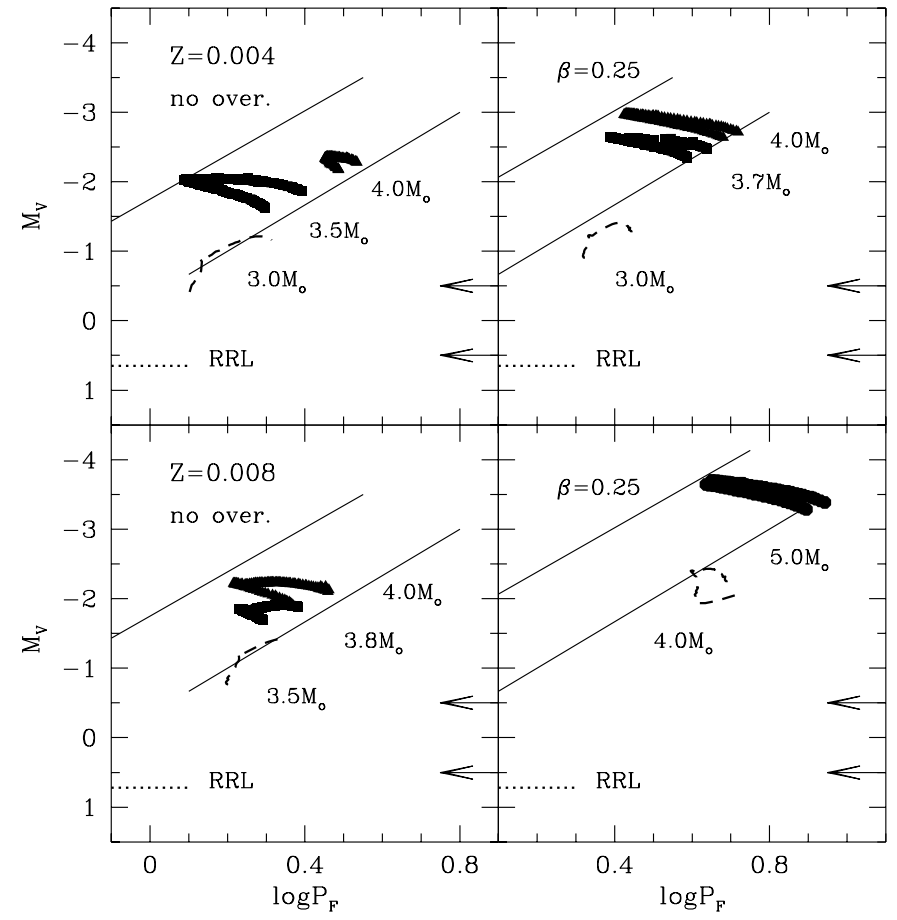

Fig. 6. As in Fig. 4, but with $Z=0.004$ and $Z=0.008$ and under different assumptions on the efficiency of overshooting. The solid lines are the same as in Fig. 4 . Note that $M_{V, \mathrm{LE}}$ is now brighter than the value predicted at $Z=0.0004$ (upper arrow in each panel), while RR Lyrae stars (dotted line) are slightly fainter (lower arrow in each panel). Moreover, the shortest period of massive pulsators ranges from $\log P_{\mathrm{F}} \sim 0.10(Z=0.004$, no overshooting) to $\sim 0.50(Z=0.008$, $\beta=0.25)$, which is significantly longer than the value $\left(\log P_{\mathrm{F}} \sim-0.3\right)$ at $Z=0.0004$.

larger than $\sim 2.0 \mathrm{mag}$ (no overshooting) or $\sim 3.0 \mathrm{mag}(\beta=0.25$ ), against the value of $\sim 1$ mag at $Z=0.0004$.

As for mass loss, we have already presented evidence that for structures with $M$ in the range 1-2 $M_{\odot}$ the post He-flash evolution is little affected by mass loss during the RGB phase. The effects of mass loss in more massive structures which quietly ignited He-burning $\left(\sim 3-4 M_{\odot}\right)$ has already been exhaustively discussed in the literature (see, e.g., Bertelli et al. 1989) and one finds that in a metal poor $4 M_{\odot}$ model even a huge amount of mass loss $\left(\sim 0.13 M_{\odot}\right)$ decreases the luminosity of the He burning blue loop by less than $0.1 \mathrm{mag}$ (Castellani et al. 2003), with little effect on the predicted pulsational scenario.

Figure 7 summarizes the results of the whole investigation, showing the predicted limiting magnitude for massive pulsators together with the magnitude of RR Lyrae stars, both as a function of the assumed metallicity over the range $Z=0.0002$ to 0.008 . As a conclusion, we predict that, over this metallicity range, short period ( $P \leq 3$ days) central He-burning pulsators more massive than RR Lyrae stars populate a common instability strip in the $M_{V}-\log P$ plane, independent of their metal content and of the occurrence of the He flash. The only discriminating parameters appear to be the faintest magnitude and the shortest period, which become fainter and shorter, respectively, as the metal content decreases.

In this respect, the present results fully support the suggestion by Cordier et al. (2003) who explain the occurrence of 


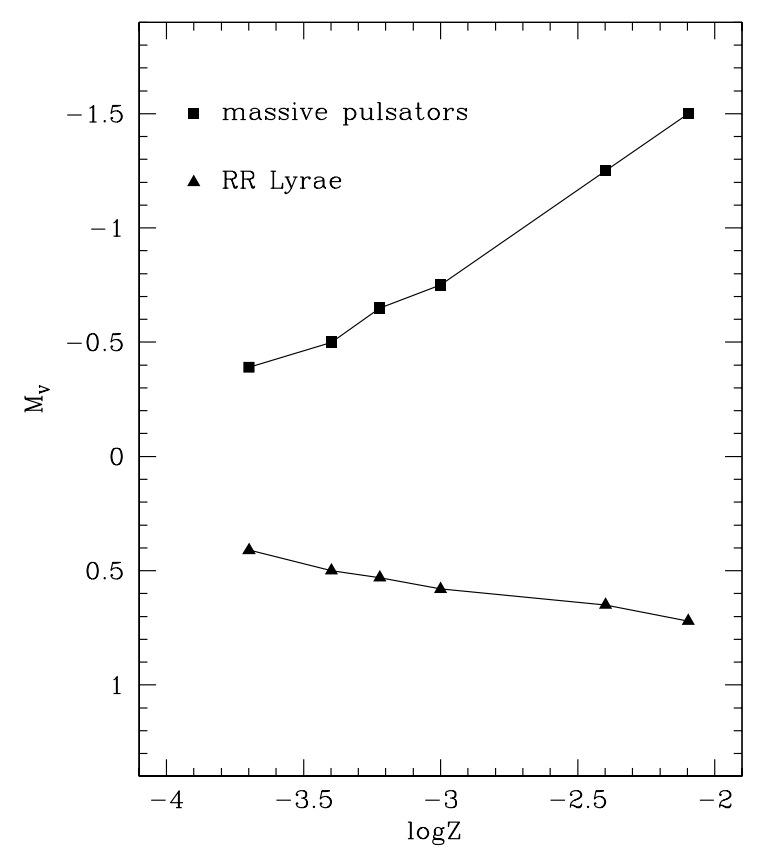

Fig. 7. The predicted limiting magnitude for massive pulsator (squares) and the magnitude of ZAHB RR Lyrae variables (triangles) as a function of the star metallicity.

faint short period Cepheids in the Small Magellanic Cloud as an effect of a decreased metallicity. Here we only add that inspection of evolutionary features for metal-deficient stars (see Cassisi et al. 1997) discloses that in the extreme case $Z=10^{-5}$ one expects pulsators with a continuous distribution of masses, with a lower mass limit at $\sim 1.0 M_{\odot}, M_{V} \sim 0.1 \mathrm{mag}$.

\section{Comparison with observations}

We can now compare the theoretical predictions with the sample of variables observed in Leo A, as given by D02 but with fundamentalised periods for FO candidates. Figure 8 shows the $M_{V}-\log P_{\mathrm{F}}$ data for variables with high quality light curves, assuming a visual distance modulus $\mu_{V}=24.6 \mathrm{mag}$ as derived from the mean magnitude $\langle V\rangle=25.1 \mathrm{mag}$ of the observed RR Lyrae stars and the predicted absolute value of 0.5 mag for $Z=0.0004$ (see Fig. 4). The solid lines depicting the instability strip in Fig. 4 are repeated here. One finds that predictions and observations appear in reasonable agreement, both for the instability edges as well as for the evidence that bright pulsators show a limiting magnitude of $\sim-0.5$ mag.

The comparison with selected evolutionary tracks shows that Cepheids in Leo A should have masses from $\sim 1.9$ to $3 M_{\odot}$, where the lower limit is the theoretical prediction for the occurrence of pulsators, whereas the upper limit only indicates the lack of more massive evolving stars. Thus the Leo A variables beautifully confirm that the distribution in the $M_{V}-\log P$ diagram of massive pulsators which experienced the He flash is the natural extension of the distribution of more massive pulsators characterized by a quiet He ignition, at least for masses $M \leq 4.0 M_{\odot}$.

As a relevant point, one finds that the discussed dependence on the metallicity appears to be supported by observational

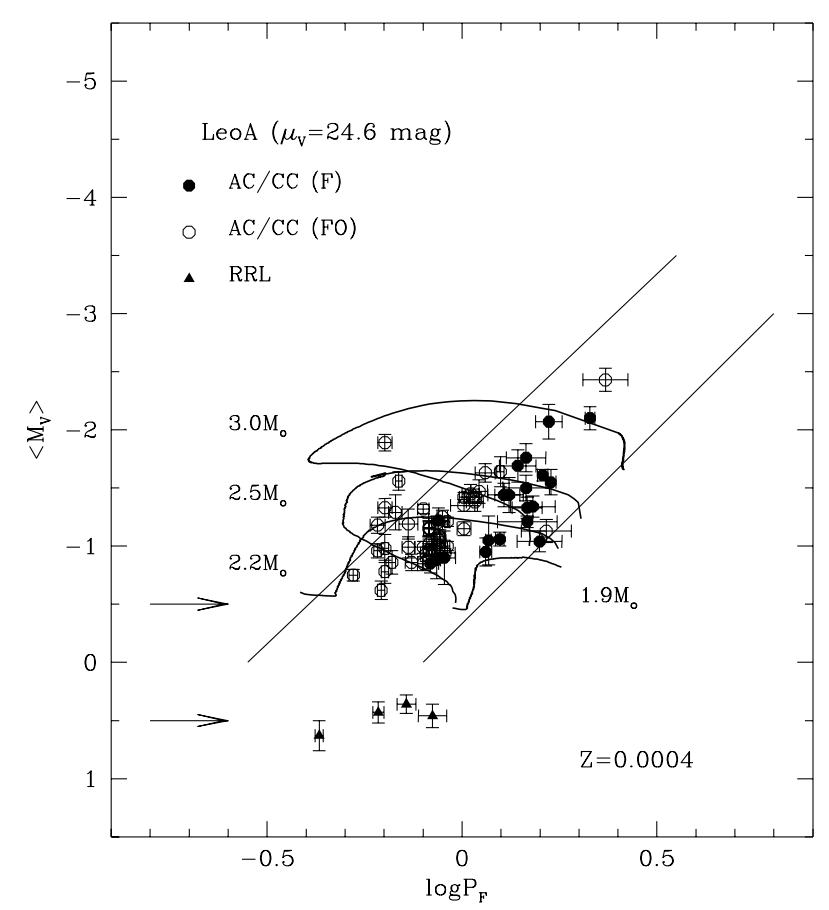

Fig. 8. Observed Cepheids (dots) and RR Lyrae stars (triangles) in Leo A as compared with the predicted edges of the instability strip in the $M_{V}-\log P_{\mathrm{F}}$ plane (solid lines). The period of first-overtone pulsators is fundamentalised. The arrows depict the theoretically predicted magnitude of the faintest Cepheids $\left(M_{V, \mathrm{LE}} \sim-0.5 \mathrm{mag}\right)$ and of RR Lyrae stars $\left(M_{V}(\mathrm{RRL}) \sim 0.5 \mathrm{mag}\right)$ at the Leo A metal content $(Z=0.0004)$. Selected evolutionary tracks are also drawn.

data. A reasonable agreement with theory is indeed obtained for Sextans A, which is one of the lowest metallicity $(Z \sim$ 0.001) galaxies with observed Classical Cepheids (see Dolphin et al. 2003). Adopting the visual distance modulus provided by the authors, we show in Fig. 9 that the absolute magnitude of the variables ranges from $M_{V} \sim-2.5 \mathrm{mag}$ to $\sim-0.75 \mathrm{mag}$, the latter being the limit predicted by theory for the metallicity of the galaxy. As shown in the figure, in this case one predicts a minimum mass of the order of $\sim 2.5 M_{\odot}$, larger than inferred from the variables in Leo A, thus suggesting that all the Cepheids in Sextans A had a quiet He-burning ignition.

Before closing this section, let us show in Fig. 10 the comparison between our predictions with $Z=0.0004$ and data for well-recognized ACs in dwarf spheroidal galaxies, using the distance moduli provided by the various authors (see Marconi et al. 2004, for references). One finds a reasonable agreement as far as the edges of the instability strip are concerned. However, if the distance moduli provided in the literature are reliable, several variables appear significantly fainter than the predicted limiting magnitude at $Z=0.0004$ ( $\sim 0.5 \mathrm{mag}$, see arrow), an occurrence which cannot be explained in terms of smaller mass at such a metal content (see Fig. 4). Moreover, the study by Marconi et al. (2004) does suggest values as low as $\sim 1.25 M_{\odot}$, as inferred by the analysis of intrinsic colors and visual amplitudes. According to the results presented in the present paper, namely the decrease of the minimum mass and luminosity with decreasing metal content, the faintest ACs plotted in Fig. 10 


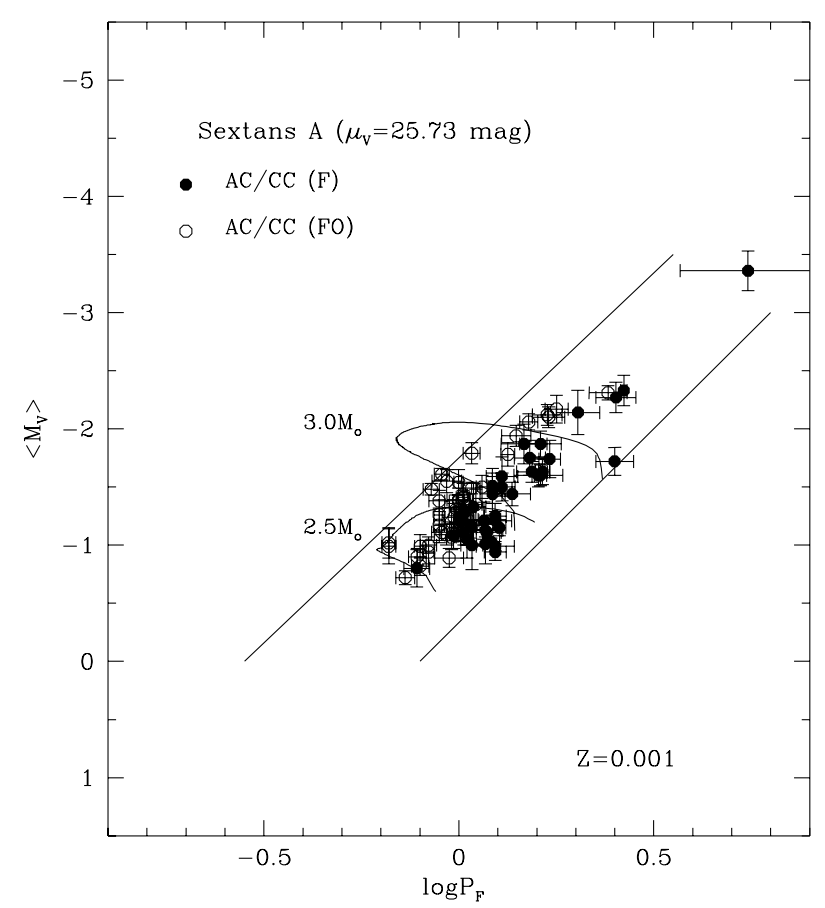

Fig. 9. As in Fig. 8, but with Classical Cepheids in Sextans A and for $Z=0.001$.

suggest the occurrence of variables with metal contents lower than $Z=0.0004$.

\section{Concluding remarks}

In the current literature, the term "Anomalous" Cepheids is widely adopted to indicate variables brighter than RR Lyrae stars and with periods $P \leq 2$ days, as repeatedly observed in dwarf spheroidal galaxies of the Local Group and, more in general, in metal-deficient stellar systems hosting not-too-old stellar populations. However, in a recent study (Dolphin et al. 2002) it has been suggested that variables with such features, as observed in the metal-poor dwarf irregular galaxy Leo A, can represent the natural extension to low metallicity of the sequence of Classical Cepheids, rather than be classified as Anomalous Cepheids.

Starting from such a suggestion, we have studied the theoretical scenario for $Z=0.0004$ to 0.008 central He-burning pulsators with mass $\leq 4 M_{\odot}$, based on updated pulsation and evolution models. Predictions for the edges of the pulsation region are derived, suggesting that $P \leq 3$ days central He-burning pulsators more massive than RR Lyrae stars populate an almost unique instability strip, independently of whether they ignited He-burning quietly or flashing, and of their metal content. The most discriminating parameter is the minimum mass for the occurrence of these bright variables, and consequently their faintest magnitude and shortest period which becomes brighter and longer when moving from $Z=0.0004$ to 0.008 .

The comparison of predictions with observed variables in Leo A $(Z \sim 0.0004)$ and Sextans A $(Z \sim 0.001)$ discloses a quite fair agreement, both for what concerns the distribution of the variables in the $M_{V}-\log P$ plane and the limiting magnitudes and periods. On this basis, present results do confirm

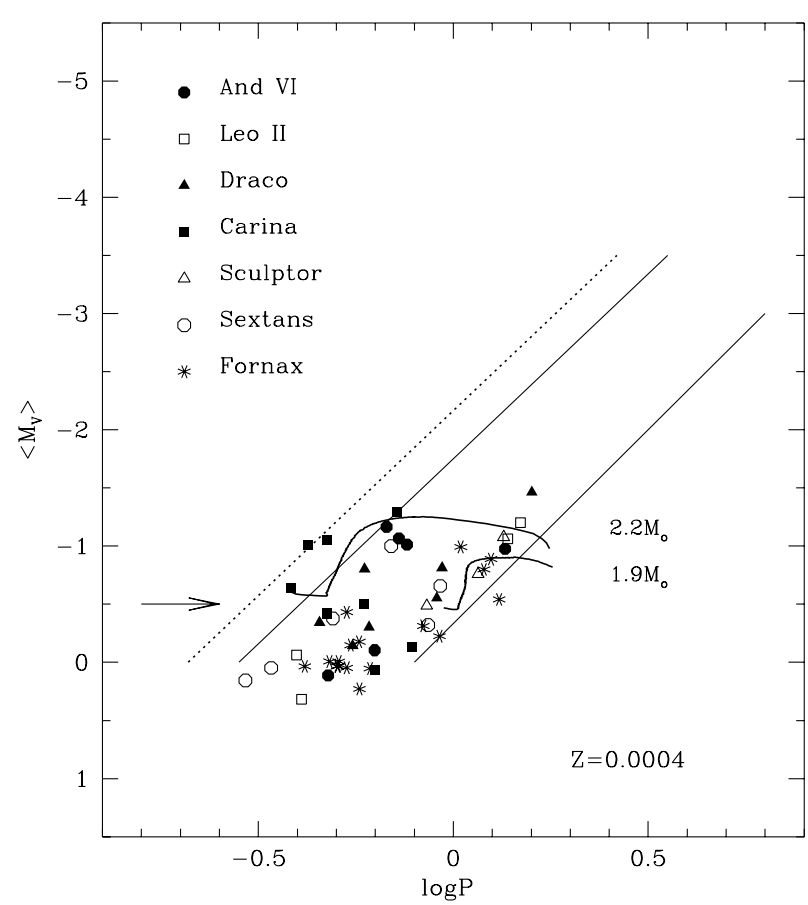

Fig. 10. Observed Anomalous Cepheids in dwarf spheroidal galaxies as compared with the predicted edges of the instability strip in the $M_{V}-\log P$ plane. The solid lines refer to the fundamental edges and are the same as in Fig. 4. To account for the occurrence of first-overtone pulsators, the blue limit is shifted by $\delta \log P=-0.13$ (dotted line). The arrow depicts the theoretically predicted magnitude of the faintest Cepheids $\left(M_{V, \mathrm{LE}} \sim-0.5 \mathrm{mag}\right)$ at $Z=0.0004$. Selected evolutionary tracks are drawn with their fundamental period.

that the so-called "Anomalous" Cepheids are the natural extension of the "Classical" Cepheids to lower metallicities, where the evolution of less massive stars intersects the pulsation region, producing pulsators with fainter magnitudes and shorter periods.

As a conclusion, not surprisingly one finds that going outside our own Galaxy the current classification of bright variables tends to be rather misleading. This is because the classical dichotomy observed in the Milky Way, old and metal-poor stellar populations against young and metal-rich ones, is vanishing. The problem we are dealing with is indeed caused by the extragalactic evidence of rather young but metal-poor populations with their pulsating instabilities. Following early discussions (see, e.g. Castellani 1986), there is now an increasing consensus in extending the term of Population II to all metal poor populations, independent of their age. In this context, there is no reason to still call "Anomalous" the massive bright pulsators observed in metal-deficient stellar systems. Wishing to retain the term "Cepheids" for pulsators brighter than RR Lyrae stars, and considering that the only distinctive factor is the pulsator mass, it wight be better to make clear the evolutionary status of the variables, using the term "HB Cepheids" (HBC) for low mass Cepheids with ZAHB progenitors (Population II Cepheids in the current nomenclature), whereas the so called "Anomalous" Cepheids should be simply regarded as bona fide metal-poor, low-mass Classical Cepheids. 
Finally, even though detailed synthetic simulations are needed to account for the stellar evolutionary lifetimes, we suggest that the observed faintest Cepheids could yield a straightforward distance estimate to the hosting stellar system, provided that no significant metallicity dispersion is present, the metal content is well-known and no completeness problem affects the Cepheid sample. We should add that, since the RR Lyrae luminosity decreases with increasing metallicity, the observed difference in magnitude between the faintest Cepheids and RR Lyrae stars with similar metal content is predicted to be a function of the metal content, increasing from $\sim 1 \mathrm{mag}(Z=0.0004)$ to $\sim 2.2 \mathrm{mag}$ and $3.2 \mathrm{mag}$ (at $Z=0.008$ without overshooting and with $\beta=0.25$, respectively), thus providing a useful metal content indicator.

Acknowledgements. We warmly thank Giuseppe Bono and Steven Shore for useful suggestions and a careful reading of the manuscript. We also thank an anonymous referee for his/her useful comments which have considerably improved the paper. Financial support for this work was provided by the scientific project "Stellar Populations in Local Group Galaxies" from MIUR-Cofin 2002 (P.I. Monica Tosi). Model computations made use of resources granted by the "Consorzio di Ricerca del Gran Sasso", according to Project 6 "Calcolo Evoluto e sue Applicazioni (RSV6) - Cluster C11/B"

\section{References}

Baldacci, L., Matonti, F., Rizzi, L., et al. 2004, MmSAI, 75, 126 Bertelli, G., Chiosi, C., \& Bertola, F. 1989, ApJ, 339, 889
Bono, G., Caputo, F., Castellani, V., \& Marconi, M. 1997a, A\&AS, 121,327

Bono, G., Caputo, F., Santolamazza, P., Cassisi, S., \& Piersimoni, A. 1997b, AJ, 113, 2209

Bono, G., Caputo, F., Castellani, V., \& Marconi, M. 1999, ApJ, 512, 711

Bono, G., Groenewegen, M. A. T., Marconi, M., \& Caputo, F. 2002, ApJ, 574, 33

Caputo, F., \& Degl'Innocenti, S. 1995, A\&A, 298, 833

Cariulo, P., Degl'Innocenti, S., \& Castellani, V. 2004, A\&A, in press

Cassisi, S., Castellani, M., \& Castellani, V. 1997, A\&A, 317, 108

Castellani, V. 1986, Progress of Cosmic Physics, 9, 317

Castellani, V., \& Degl'Innocenti, S. 1995, A\&A, 298, 827

Castellani, V., Degl'Innocenti, S., Marconi, M., Prada Moroni, P. G., \& Sestito, P. 2003, A\&A, 404, 645

Castelli, F., Gratton, R., \& Kurucz, R. 1997, A\&A, 318, 841

Chiosi, C., Wood, P. R., \& Capitanio, N. 1993, ApJS, 86, 541

Cordier, D., Goupil, M. J., \& Lebreton, Y. 2003, A\&A, 409, 491

Da Costa, G. S., Armandroff, T. E., \& Caldwell, N. 2002, AJ, 124, 332

Dall'Ora, M., Ripepi, V., Caputo, F., et al. 2003, AJ, 126, 197

Di Criscienzo, M., Marconi, M., \& Caputo, F. 2004, ApJ, submitted

Dolphin, A., Saha, A., Claver, J., et al. 2002, AJ, 123, 3154 (D02)

Dolphin, A., Saha, A., Skillman, E. D., et al. 2003, AJ, 125, 1261

Marconi, M., Caputo, F., Di Criscienzo, M., \& Castellani, M. 2003, ApJ, 596, 299

Marconi, M., Fiorentino, G., \& Caputo, F. 2004, A\&A, 417, 1101

Mateo, M. 1998, ARA\&A, 36, 435

Pritzl, B., Armandroff, T., Jacoby, G., \& Da Costa, G. 2002, AJ, 124, 1464

Siegel, M. H., \& Majewski, S. R., 2000, AJ, 120, 284

Wallerstein, G. 2002, PASP, 114, 689 